\begin{abstract}
It is shown from elementary considerations that polarised beams of electrons or positrons are unlikely to be obtained in storage rings much above about $30 \mathrm{GeV}$ in top energy.

Normal design criteria in such machines result in large energy spreads which straddle depolarising resonances. The requirement that such resonances be weak enough as not to cause substantial depolarisation, imposes control of vertical closed-orbit errors and beam size beyond what is currently believed to be feasible.
\end{abstract}

$$
\text { Geneva - June, } 1976
$$


1.

Radiative polarisation of electrons and positrons, predicted by Sokolov and Ternov 1 ) and observed in ACO 2), VEPP-2 3) and SPEAR 4), is in competition with various depolarising effects. To obtain, and maintain, a high degree of polarisation in the beam requires that the depolarisation time $\tau_{\text {dep }}$ be much greater than the polarisation time $\tau_{\text {pol }}$ ' given by

$$
\tau_{\mathrm{pol}}=\frac{8 \mathrm{~m}_{0} \mathrm{R} \rho^{2}}{5 \sqrt{3} \mathrm{~h} r_{0} \gamma^{5}}
$$

where $R$ is the average radius and $\rho$ the bending radius of the machine, $r_{0}$ the classical radius of the electron, $n$ the normalised Planck's constant, $\mathrm{m}_{0}$ the electron rest mass and $\gamma$ the Lorentz factor.

Typical polarisation times at top energy: range from a few tens of minutes to several hours. The Large Electron Positron storage ring 5 ) (LEP), currently under study at CERN, has $\tau_{\text {pol }} \approx 50$ minutes at $100 \mathrm{GeV}$. At lower energy in a given machine $\tau_{\text {pol }}$ would increase rapidly unless wigglers were used to prevent this ${ }^{6)}$.

Depolarisation can occur if particles spend an appreciable fraction of their time in the neighbourhood of spin resonances. Such resonances occur for

$$
\gamma a=k \pm k_{x} Q_{x} \pm k_{z} Q_{z} \pm k_{s} Q_{s}
$$

where $\mathrm{a}=\frac{\mathrm{g}-2}{2} \approx \frac{\alpha}{2 \pi} \approx 1.16 \times 10^{-3}, \mathrm{~g}$ is the gyromagnetic ratio, $\alpha$ the fine-structure constant, $k, k_{x}, k_{z}, k_{s}$ are positive integers (or zero), $Q_{x}, Q_{z}$ the betatron wave numbers and $Q_{s}$ the synchrotron oscillation wave number.

Strong resonances, known as imperfection resonances, are those given by $\gamma a=k$, and correspond to an integral number of spin-precessions per revolution of the machine. They are driven mainly by radial fields 
associated with the $\mathrm{k}^{\prime}$ th harmonic of the vertical closed-orbit distortion through imperfections and focusing forces, which produce resonant precession perturbations. In addition, longitudinal fields at magnet ends can influence the resonance strength; however, we consider here only the radial field effects, which are likely to be the dominant ones in high energy machines.

\section{SPACING OF RESONANCES}

The condition $\gamma a=k$ gives immediately the energy spacing of adjacent resonances

$$
\Delta \mathrm{E}=\frac{\mathrm{m}_{0} \mathrm{c}^{2}}{\mathrm{a}} \approx 440 \mathrm{MeV}
$$

which is determined only by fundamental constants. (A similar relation "wàs used by Hereward 7) for protons.) This is the maximum space available tolaccommodate the natural energy spread of the electron bunches, but the presence of other resonance families may reduce this further. The ständard deviation $\sigma_{\mathrm{E}}$ of the energy spread in an electron storage ring is given approximately by

$$
\sigma_{E}=\left(\frac{55 \hbar c m_{0} c^{2}}{32 \sqrt{3}}\right)^{\frac{1}{2}} \frac{\gamma^{2}}{\sqrt{\rho}}
$$

In the lower energy range $\sigma_{\mathrm{E}}$ is sufficiently small to be accommodated comfortably between two adjacent spin resonances. For example, in the electron-proton option of the CERN Large Storage Ring design (LSR) 8) the $20 \mathrm{GeV}$ electrons have $\sigma_{\mathrm{E}} \approx 15 \mathrm{MeV}$, which provides about $\pm 14 \sigma_{\mathrm{E}}$ between the $\gamma a=k$ resonances. In contrast, LEP at:100. GeV has

$\sigma_{E} \approx 110 \mathrm{MeV}$, leaving only $\pm 2 \sigma_{E}$ spacing. Thus, with the nominal energy centred between two such resonances, the fraction of the beam outside $2 \sigma_{E}$ crosses these resonances four times in each period of synchrotron oscillation, and rapid depolarisation might occur. 
3.

LOWER LIMIT OF $\tau_{\text {dep }}$

This can be obtained by analogy with the quantum lifetime $\tau_{2} \sigma$ for an energy-aperture limitation at $\pm 2 \sigma_{E}$, which is a measure of the diffusion rate at this part of the Gaussian distribution. If the particles outside $2 \sigma_{E}$ were fully depolarised within a time $\tau_{2} \sigma$ due to the repeated resonance crossing, then $\tau_{\text {dep }} \approx \tau_{2 \sigma}$. Since $\tau_{2 \sigma}$ is about one radiation damping time, $\sim 12 \mathrm{msec}$ for $L E P$, the beam would never develop any measurable polarisation if this situation were to occur in practice. To examine this further we consider in more detail the crossing of the resonances.

4. RESONANCE WIDTH

We assume that the spin perturbation arises from the radial magnetic field $B_{r}(t)$ associated with the $k^{\prime}$ th Fourier component of a vertical closed-orbit imperfection

$$
z=z_{k} \sin k \Omega_{r} t
$$

where $\Omega_{r}=\frac{C}{R}$ is the orbit revolution frequency, $R$ being the average radius. The corresponding field component is

$$
B_{Y}(t)=-\frac{m_{0} c \gamma k^{2} z_{k}}{e R^{2}} \sin k \Omega_{r} t=B_{k} \sin k \Omega_{r} t
$$

and the precession perturbation frequency 9) is (for ya $\gg 1$ )

$$
\omega_{k}(t)=-\frac{e a}{m_{0}} B_{r}(t)=\frac{c \gamma a k^{2} z_{k}}{R^{2}} \sin k \Omega_{r} t
$$

The resonance width $\hat{\omega}$ is obtained by averaging the product of $\omega_{k}(t)$ and a phase term which is modulated with the superperiodicity of the machine. However, apart from certain systematic resonances we can write to within a factor of order unity

$$
\hat{\omega} \approx \frac{\mathrm{c} \gamma_{\mathrm{a} k^{2} z_{\mathrm{k}}}}{\mathrm{R}^{2}}
$$


We express this resonance width in terms of the corresponding energy width

$$
[\Delta \gamma]_{\text {res }}=\frac{\hat{\omega}}{a \Omega_{r}}=\frac{\gamma k^{2} z_{k}}{R}=\frac{(\gamma a)^{3}}{a}-\frac{z_{k}}{R}
$$

(since we are considering the resonance $\mathrm{ra}=\mathrm{k}$ ).

In order that the $k^{\prime}$ th and $(k+1)^{\prime}$ th resonances be distinct and well separated, we must have the condition

$$
\mathrm{m}_{0} \mathrm{c}^{2}[\Delta \gamma]_{\text {res }} \ll \frac{\mathrm{m}_{0} \mathrm{c}^{2}}{\mathrm{a}} \quad(\text { from }(2))
$$

so, from (6),

$$
z_{k} \ll \frac{R}{(\gamma a)^{3}}
$$

Now for LEP at $100 \mathrm{GeV}, \gamma \approx 2 \times 10^{5}, \mathrm{R}=8.1 \mathrm{~km}, \mathrm{~B}_{0}=546$ gauss and $\rho=6.1 \mathrm{~km}$. Then $\gamma a=232$ and the criterion (7) for distinct, separated resonances is

$$
\mathrm{z}_{\mathrm{k}} \ll 0.65 \mathrm{~mm} \text {, or } \mathrm{B}_{\mathrm{k}} \ll 2 \text { gauss }
$$

If this is not satisfied, a large fraction of the particles will be living permanently inside the resonances and no polarisation is possible. Note that (7) is a neccessary but not a sufficient condition for polarisation to be possible.

\section{RESONANCE CROSSING SPEED}

Particles may cross a spin resonance either smoothly, as a result of synchrotron (energy) oscillations, or stochastically as a consequence of quantum jumps in energy. For the smooth motion two limiting regimes can be distinguished, "fast" crossing and "slow" crossing 9,10). In a fast crossing, the perturbation of the spin precession is very small during the particles' passage through the resonance and the polarisation of the beam is only slightly reduced by each traversal. Slow crossings are characterised by the perturbed precession 
motion varying very slowly and adiabatically on the scale of the unperturbed precession frequency. If the resonance traversal takes place strictly symmetrically either side, almost the whole population undergoes a spin flip and the polarisation of the beam is reversed 9 ).

A parameter characterising the crossing speed is 3 )

$$
\begin{aligned}
\kappa=\left[\frac{\pi \hat{\omega}^{2}}{2 a \Omega_{r} \frac{d \gamma}{d t}}\right] & =\frac{(\gamma a)^{3} z_{k}}{R}\left[\frac{\pi c}{2 a R \frac{d \gamma}{d t}}\right]^{\frac{1}{2}} \\
& =\frac{\gamma a B_{k}}{B_{0}}\left[\frac{\pi c}{2 a R \frac{d \gamma}{d t}}\right]^{\frac{1}{2}}
\end{aligned}
$$

where we have expressed $k$ also in terms of the $k^{\prime}$ th harmonic of the radial field from Eq. (4) and of the bending field $B_{0}$. The criteria are

$$
\begin{aligned}
& \kappa \ll 1 \text { for fast crossing } \\
& k \gg>1 \text { for slow crossing }
\end{aligned}
$$

If we take as reference those particles executing synchrotron oscillations at frequency $\Omega_{S}$ and amplitude $\sigma_{E}$, the maximum value of $\frac{d \gamma}{d t}$ is

$$
\left(\frac{d \gamma}{d t}\right)_{\max }=\frac{\Omega_{S} \sigma_{E}}{m_{0} c^{2}}
$$

which, for LEP, is $\sim 0.85 \times 10^{6} \mathrm{~s}^{-1}$. This value in (8) makes the criterion for a fast crossing

$$
\mathrm{z}_{\mathrm{k}} \quad<0.08 \mathrm{~mm} \quad\left(\mathrm{~B}_{\mathrm{k}}<<0.3 \text { gauss }\right)
$$

For constant $\frac{d y}{d t}$, the polarisation before $\left(P_{0}\right)$ and after $(P)$ traversal of a resonance (in the absence of stochastic motion), were shown by Froissart and Stora 9 ) to be related by

$$
P=P_{0}\left(2 e^{-\frac{k^{2}}{4}}-1\right)
$$


We assume that the effect of repetitive crossing is mainly additive because of randomisation due to quantum energy fluctuations. For a fast crossing, the depolarisation $D$ per resonance traversal is then

$$
\mathrm{D}=\frac{\mathrm{P}_{0}-\mathrm{P}}{\mathrm{P}_{0}} \approx \frac{\mathrm{K}^{2}}{2}
$$

Taking account of the number of phase oscillations in the polarisation time, Eq. (1), one can readily estimate that the inequality (8a) must be satisfied by several orders of magnitude for appreciable polarisation to develop. Quadrupole alignment to $0.1 \mathrm{~mm} \mathrm{rms}$ might reduce $\mathrm{z}_{\mathrm{k}}$ to a few microns, but this still falls short by a considerable amount.

The slow crossing regime is only of academic interest for high energy electron rings, since it implies broad resonances and a varying distribution of precession angles across the resonance, according to initial phases. Thus, the part of the beam inside a resonance is largely depolarised, even in the idealised situation of coherent adiabatic motion.

Consideration of the energy fluctuations due to quantum emission of photons results in an even more unfavourable situation. The critical photon energy may be expressed as a fraction of the beam energy by

$$
\frac{[\Delta \gamma]_{c}}{\gamma}=\frac{\hbar \omega_{c}}{m_{0} c^{2} \gamma} \frac{3 \hbar c}{2 m_{0} c^{2}} \cdot \frac{\gamma^{2}}{\rho}
$$

For high energy electron rings this quantity is of order $10^{-6}$, i.e. about $10^{-3}$ of a standard deviation in bunch energy spread. Since the mean number of quanta emitted per electron in each machine revolution

$$
\langle\mathbf{N}\rangle=\frac{5 \pi}{\sqrt{3}} \propto \gamma
$$

is around $10^{4}$ for these large machines, the diffusion rate is fast on the scale of synchrotron oscillation period, and coherence of spin 
motion will be rapidly destroyed for particles crossing a resonance in the "slow" regime. Even in a low energy storage ring like ACO, depolarisation takes place in a few seconds when the beam is brought on to the resonance $\gamma a=1$ at $440 \mathrm{Mev}^{2}$ ).

\section{CONCLUSIONS}

In very high energy electron storage rings optimised according to normal criteria, the energy spread in the bunches is so large that a substantial fraction of the beam sweeps through depolarising resonances due to synchrotron (energy) oscillations.

Broad resonances and slow crossings create strong depolarising effects due to phase mixing and quantum fluctuations of energy. Narrow resonances and fast crossings, sufficient to reduce the depolarisation rate to an acceptable level, require, at $100 \mathrm{GeV}$, vertical closed orbit errors considerably smaller than the best we can envisage. Furthermore, depolarisation effects, which we have not considered in detail, arising from free vertical betatron oscillations and the proximity of resonances of type $\gamma a=k \pm k_{z} \ell_{z}$, could impose impracticable constraints on vertical beam size. Recent results at SPEAR 1l) suggest that this can be a serious problem even in the lower range of energies.

We conclude that, at $100 \mathrm{GeV}$, polarised electron or positron beams are practically excluded. The maximum energy at which polarised beams may be feasible is unlikely to be much above $30 \mathrm{GeV}$, at which energy the depolarisation time might be comparable to the quantum lifetime for a $6 \sigma$ aperture. The energy may even be lower when all the potential sources of depolarisation are taken into account. 


\section{REFERENCES}

1) A.A. Sokolov and I.M. Ternov; Sov. Phys. Doklady, 8,1203 (1964).

2) The Orsay Storage Ring Group; Proc. VIIIth Int. Conf. on High Energy Accelerators, CERN (1971) p. 127.

3) V.N. Baier; Sov. Phys. Uspekhi, 14, 695 (1972).

4) U. Camerini, D. Cline, J. Learned, A.K. Mann, L.K. Resvanis; Univ. of Pennsylvania preprint (July 1975).

5) B. Richter; Report CERN/ISR-LTD/76-9 (March 1976). (Talk delivered at the Meeting on $\mathrm{e}^{+} \mathrm{e}^{-}$Physics, Flaine, Haute-Savoie, February $22-28$, 1976).

6) A. Hutton; Report CERN/ISR-LTD/76-18 (May 1976). (Submitted to Particle Accelerators).

7) H.G. Hereward; Proc. Summer Studies on High Energy Physics with Polarised Beams, Argonne Nat. Lab. Rpt. ANL/HEP 75-02, p XVII, 1 (1975).

8) B.W. Montague; Report CERN/ISR-LTD/76-16 (April 1976). (Talk delivered at the Meeting on $\mathrm{e}^{+} \mathrm{e}^{-}$Physics, Flaine, Haute-Savoie, February $22-28$, 1976).

9) M. Froissart and R. Stora; Nucl. Inst. and Methods, 7, 297 (1960).

10) Ya.S. Derbenev, A.M. Kondratenko and A.N. Skrinsky; Sov. Phys. JETP, 33, 658 (1971).

11) A.W. Chao and R.F. Schwitters; private communication (May 1976). 
a i $1: \quad \because \quad \therefore$

? : : :

: $1:$

ง. $\because$.

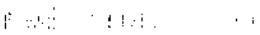

$\begin{array}{lll}3 & 01\end{array}$

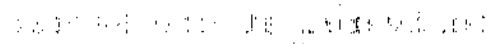

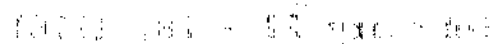

